\title{
Performance enhancement of wireless sensor network by using non-orthogonal multiple access and sensor node selection schemes
}

\author{
Duy Hung $\mathrm{Ha}^{1}$, Dac-Binh $\mathrm{Ha}^{2}$, Van-Truong Truong ${ }^{3}$, Van-Duc Phan ${ }^{4}$, Q. S. Vu ${ }^{5}$ \\ ${ }^{1}$ Wireless Communications Research Group, Faculty of Electrical and Electronics Engineering, Ton Duc Thang \\ University, Ho Chi Minh City, Vietnam \\ ${ }^{2,3}$ Faculty of Electrical-Electronic Engineering, Duy Tan University, Da Nang, Vietnam \\ ${ }^{4}$ Faculty of Automobile Technology, Van Lang University, Ho Chi Minh City, Vietnam \\ ${ }^{5}$ School of Engineering-Technology \& Arts, Hong Bang International University, Ho Chi Minh City, Vietnam
}

\begin{tabular}{l} 
Article Info \\
\hline Article history: \\
Received Jun 4, 2020 \\
Revised Aug 3, 2020 \\
Accepted Aug 11, 2020 \\
\hline
\end{tabular}

\section{Keywords:}

Amplify-and-forward

Best sensor node selection

NOMA

Outage probability

Throughput

WSN
This is an open access article under the CC BY-SA license.

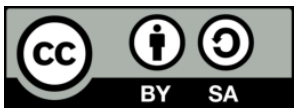

\section{Corresponding Author:}

Dac-Binh Ha

Faculty of Electrical-Electronic Engineering

Duy Tan University, Da Nang, Vietnam

Email: hadacbinh@duytan.edu.vn

\section{INTRODUCTION}

In the last decades, wireless sensor networks (WSN) have been widely applied in many fields, such as monitoring environmental parameters in industry and agriculture, smart transport, smart grids, wearable medical care devices [1-3]. The main advantage of wireless sensor networks is that the use of existing infrastructure may not incur additional costs of wiring and equipment, and further, the cloud availability and IoT protocol for a fast connection. However, WSN also contains many problems that need to be addressed, typically data transmission between devices in the network when the scale of the network becomes very large with a massive amount of data [4]. The non-orthogonal multiple access (NOMA) technique was proposed as the best solution to solve the above problem when satisfying the very high data rate and massive connectivity demand both in uplink and downlink transmissions [5-7]. NOMA can support multiple users at the same time and the same frequency resource. In WSN, the uplink channel plays a critical role, as this is the path that the sensors used to perform the task of transmitting the data they collect to the sink node. However, studies on WSN using uplink NOMA are still relatively small [8-12]. In [8], the authors proposed WSN to use the uplink NOMA application to measure parameters in agriculture. The sensors are divided into clusters and use short-range multi-hop communication technology to transfer data to the sink node. The authors used the sum 
data rate and outage probability to examine the performance of the system. In [9], the uplink NOMA multiuser model was proposed.

The base station is equipped with $\mathrm{N}$ antennas, and each user equipment unit has a single antenna, the users are divided into two groups: robust set and weak set depending on the channel status. The paper proposes that the power control scheme can maximize the sum capacity with a minimum target rate. Another common problem with WSN is the transmission distance in the network. The sensor nodes located at the network edge can only communicate with neighboring nodes and need support from the relay to communicate with the sink node. Transition techniques combined with NOMA helps solve this problem in WSN [13-25]. In [13], the authors proposed the uplink NOMA model for two users with the support of the decode-and-forward relay to communicate with the base station. The paper goes into system analysis by providing formulas for system probability and throughput based on critical parameters such as signal to noise ratio, transmission power. In [16], two users communicated with the base station simultaneously with the existence of a half-duplex relay employing the decode-and-forward scheme to assist the far user. With the given target data rates, the authors proposed the method to determine the most optimal power allocation factors.

Different from previous studies, in this study, we propose a NOMA scenario for the uplink of two sensor node clusters, in which the two sensors apply the uplink NOMA scheme to transmit their information to the sink via the AF relay. The proposed model uses the best combination of direct link and forward-link selection mechanism based on the maximum signal to end-to-end noise ratio. To analyze the performance of this system, we derive expressions of the outage probability and throughput by using the GaussianChebyshev quadratic. The numerical results will be calculated according to the main parameters: transmission power, the number of sensor nodes to find ways to improve the performance of this system.

The remainder of this research can be formulated as follows. Section 2 presents the system model. Section 3 analyzes the system performance. Section 4 shows the numerical results with some discussions. Finally, Section 5 concludes the study.

\section{SYSTEM MODEL}

We consider an uplink NOMA relaying system for WSN as Figure 1. This system consists of two sensor clusters $\mathrm{P}$ with $\mathrm{N}$ sensor nodes and $\mathrm{Q}$ with $\mathrm{M}$ sensor nodes, a relay node, and a sink. Two sensor nodes selected respectively from $\mathrm{P}$ and $\mathrm{Q}$ transmit their messages to the sink node (S) with the help of a single AF R. Assuming that the sensing data, i.e., confidential data, video data, from $\mathrm{P}$ is more important than the data, i.e., humidity, temperature, from Q. Therefore, the best sensor node is selected from $\mathrm{P}$ (SNP*), meantime, the sensor node of Q (SNQ) is randomly chosen to transmit their data to S via R. All sensor nodes, relay, and sink have single-antenna and work in half-duplex mode. Suppose R has sufficient channel information from the sensor cluster, thus according to the maximum channel power gain, $\mathrm{R}$ assigns a sensor node as the best node SNP* from $\mathrm{P}$ to send sensing data to it.

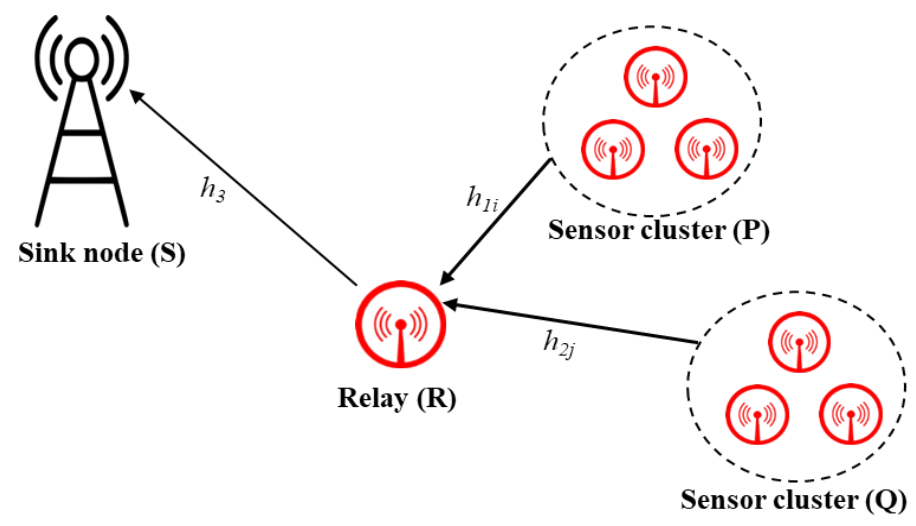

Figure 1. System model

The proposed operating protocol for this system is given as follows:

Phase 1: SNP* and SNQ simultaneously transmit their signals (s1, s2) to R during the period of T/2, where T denotes as transmission block time. 
Phase 2: During the remaining $\mathrm{T} / 2$ period, $\mathrm{R}$ amplifies and forwards the signal received from $\mathrm{SNP}^{*}$ and SNQ to S. Finally, S uses the successive interference cancellation (SIC) to detect the signal of SNP* and SNQ to obtain the information of each node.

We describe in detail each phase mathematically as follows.

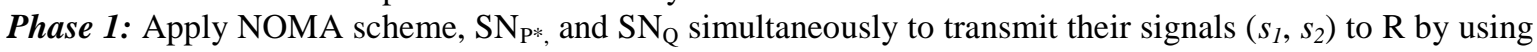
their transmit power on the same frequency in the same period $\mathrm{T} / 2$.

The signal received at $\mathrm{R}$ has the following form:

$$
y_{1}=\sqrt{\frac{P_{1}}{d_{1}^{\theta}}} h_{1} s_{1}+\sqrt{\frac{P_{2}}{d_{2}^{\theta}}} h_{2} s_{2}+n_{1},
$$

where $h_{k}(k=\{1,2\})$ are Rayleigh fading channel coefficients of links from $\mathrm{SN}_{\mathrm{P}^{*}}$ and $\mathrm{SN}_{\mathrm{Q}}$ to $\mathrm{R}$, respectively, $n_{1}$ is AWGN with zero mean and the variance of $\sigma^{2}, n_{2} \sim C N\left(0, \sigma^{2}\right), d_{1}$ and $d_{2}$ are the Euclidean distances of $\mathrm{SN}_{\mathrm{P} *}$ and $\mathrm{SN}_{\mathrm{Q}}$ to $\mathrm{R}$, respectively, and $\theta$ represents the path-loss exponent.

Phase 2: Applying the AF scheme, the transmission signal at R has the transmission power $P_{R}$ can be formulated by,

$$
y_{R}=G y_{1} \text {. }
$$

In particular, $G$ is the relaying gain of the $\mathrm{AF}$ relay $\mathrm{R}$, which is defined by the fact that the $P_{3}$ bounds the transfer power of the relay. Therefore, $G$ is given by,

$$
G=\sqrt{\frac{P_{3}}{P_{1}\left|h_{1}\right|^{2} / d_{1}^{\theta}+P_{2}\left|h_{2}\right|^{2} / d_{2}^{\theta}+\sigma^{2}}} .
$$

Therefore, the signal received at $\mathrm{S}$ is written as,

$$
y_{2}=\frac{G h_{3}}{\sqrt{d_{3}^{\theta}}}\left(\sqrt{\frac{P_{1}}{d_{2}^{\theta}}} h_{1} s_{1}+\sqrt{\frac{P_{2}}{d_{2}^{\theta}}} h_{2} s_{2}+n_{1}\right)+n_{2}
$$

where $h_{3}$ and $d_{3}$ are the Rayleigh fading channel coefficient and Euclidean distance of $\mathrm{R}$ and S, respectively $n_{2} \sim C N\left(0, \sigma^{2}\right)$

Finally, $\mathrm{S}$ applies SIC to detect the signals of $\mathrm{SN}_{\mathrm{P} *}$ and $\mathrm{SN}_{\mathrm{Q}}$. The process is as follows: $s_{1}$ will be detected first due to better channel condition, then separation of signal $s_{2}$ by subtracting $s_{1}$ from $y_{2}$. The instantaneous SINR for detecting $s_{l}$ at $\mathrm{S}$ is given by,

$$
\begin{aligned}
\gamma_{s_{1}} & =\frac{P_{1} G^{2}\left|h_{1}\right|^{2}\left|h_{3}\right|^{2} / d_{1}^{\theta} d_{3}^{\theta}}{G^{2}\left(P_{2}\left|h_{2}\right|^{2} / d_{2}^{\theta}+\sigma^{2}\right)\left|h_{3}\right|^{2} / d_{3}^{\theta}+\sigma^{2}} \\
& =\frac{\gamma_{1} \gamma_{3}\left|h_{1}\right|^{2}\left|h_{3}\right|^{2}}{\gamma_{2} \gamma_{3}\left|h_{2}\right|^{2}\left|h_{3}\right|^{2}+\gamma_{1}\left|h_{1}\right|^{2}+\gamma_{2}\left|h_{2}\right|^{2}+\gamma_{3}\left|h_{3}\right|^{2}+1},
\end{aligned}
$$

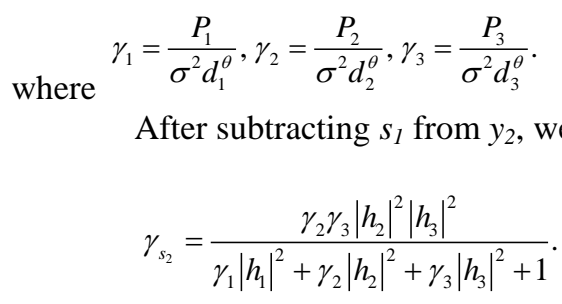

The Probability Density Function (PDF) and Cumulative Distribution Function (CDF) functions of the random variable $X_{i}(i=\{1,2\})$ are calculated as, respectively, 


$$
\begin{aligned}
& f_{X_{i}}(x)=\frac{1}{\lambda_{i}} e^{-\frac{x}{\lambda_{i}}} . \\
& F_{X_{i}}(x)=1-e^{-\frac{x}{\lambda_{i}}} .
\end{aligned}
$$

Meanwhile, due to selecting the best channel of links P-R, the CDF and PDF of channel power gain $Y_{1}=\max _{1 \leq i \leq N}\left\{\left|h_{1 i}\right|^{2}\right\}$ is given as follows,

$$
\begin{aligned}
& F_{Y_{1}}(x)=\left(1-e^{-\frac{x}{\lambda_{1}}}\right)^{N}=\sum_{i=0}^{N}\left(\begin{array}{c}
N \\
i
\end{array}\right)(-1)^{i} e^{-\frac{i x}{\lambda_{1}}} \\
& f_{Y_{1}}(x)=\frac{N}{\lambda_{1}} e^{-\frac{x}{\lambda_{1}}}\left(1-e^{-\frac{x}{\lambda_{1}}}\right)^{N-1}=\frac{N}{\lambda_{1}} \sum_{i=0}^{N-1}\left(\begin{array}{c}
N-1 \\
i
\end{array}\right)(-1)^{i} e^{-\frac{(i+1) x}{\lambda_{1}}} .
\end{aligned}
$$

For the later calculation, we obtain the expression of CDF for $U_{1}=\frac{\gamma_{1} Y_{1}}{\gamma_{2} X_{2}+1}$ as follows:

$$
\begin{aligned}
& F_{U_{1}}(x)=\operatorname{Pr}\left(\frac{\gamma_{1} Y_{1}}{\gamma_{2} X_{2}+1}<x\right)=1+\frac{\lambda_{1} \gamma_{1}}{\lambda_{1} \gamma_{1}+i \lambda_{2} \gamma_{2} x} \sum_{i=1}^{N}\left(\begin{array}{c}
N \\
i
\end{array}\right)(-1)^{i} e^{-\frac{i x}{\lambda_{1} \gamma_{1}}} \\
& f_{U_{1}}(x)=\sum_{i=1}^{N}\left(\begin{array}{c}
N \\
i
\end{array}\right)(-1)^{i+1}\left(\frac{\lambda_{1} \gamma_{1} \lambda_{2} \gamma_{2}}{\left(\lambda_{1} \gamma_{1}+i \lambda_{2} \gamma_{2} x\right)^{2}}+\frac{1}{\lambda_{1} \gamma_{1}+i \lambda_{2} \gamma_{2} x}\right) i e^{-\frac{i x}{\lambda_{1} \gamma_{1}}} .
\end{aligned}
$$

\section{PERFORMANCE ANALYSIS}

The outage probability $P_{\text {out }}$ for detection of $s_{1}$ and $s_{2}$ are expressed as follows, respectively:

$$
\begin{aligned}
& P_{\text {out }}^{s_{1}}=\operatorname{Pr}\left(\gamma_{s_{1}}<2^{2 \Omega}-1=\gamma_{t}\right) \\
& P_{\text {out }}^{s_{2}}=\operatorname{Pr}\left(\gamma_{s_{2}}<\gamma_{t}\right)
\end{aligned}
$$

\section{Theorem 1.}

Under quasi-static Rayleigh fading, the closed-form expression of $P_{\text {out }}$ to detect $s_{l}$ for this considered system is given by,

$$
\begin{gathered}
P_{\text {out }}^{s_{1}}=1+\frac{\pi \mu}{2 L} \sum_{i=1}^{N}\left(\begin{array}{c}
N \\
i
\end{array}\right)(-1)^{i} i e^{-\left(\frac{i}{\lambda_{1} \gamma_{1}}+\frac{1}{\lambda_{3} \gamma_{3}}\right) \gamma_{t}} \sum_{l=1}^{L}\left[\frac{\lambda_{1} \gamma_{1} \lambda_{2} \gamma_{2} \alpha_{l}+\lambda_{1} \gamma_{1} \alpha_{l}+i \lambda_{2} \gamma_{2} \gamma_{t} \alpha_{l}-i \lambda_{2} \gamma_{2} \mu}{\left(\lambda_{1} \gamma_{1} \alpha_{l}+i \lambda_{2} \gamma_{2} \gamma_{t} \alpha_{l}-i \lambda_{2} \gamma_{2} \mu\right)^{2} \alpha_{l}}\right] e^{\frac{i \mu}{\lambda_{1} \gamma_{1} \alpha_{l}}} \sqrt{1-a_{l}^{2}}, \\
\alpha_{l}=\ln \frac{a_{l}+1}{2}, \quad \mu=\frac{\gamma_{t}\left(\gamma_{t}+1\right)}{\lambda_{3} \gamma_{3}}, \quad a_{l}=\cos \left(\frac{2 l-1}{2 L} \pi\right) .
\end{gathered}
$$

Proof: See Appendix A.

\section{Theorem 2}

Using quasi-static Rayleigh fading, the closed-form expression of the outage probability to detect $s_{2}$ for this considered system is given by 


$$
P_{\text {out }}^{s_{2}}==1-\frac{\pi \mu^{2} N \lambda_{3} \gamma_{3}}{2 L \lambda_{2} \gamma_{2}} e^{-\left(\frac{1}{\lambda_{2} \gamma_{2}}+\frac{1}{\lambda_{3} \gamma_{3}}\right) \gamma_{t}} \sum_{i=0}^{N-1}\left(\begin{array}{c}
N-1 \\
i
\end{array}\right)(-1)^{i} \sum_{l=1}^{L} \frac{e^{\frac{\mu}{\lambda_{2} \gamma_{2} \alpha_{l}}} \sqrt{1-a_{l}^{2}}}{\alpha_{l}^{2}\left[\lambda_{1} \gamma_{1} \gamma_{t} \alpha_{l}-(i+1) \lambda_{3} \gamma_{3} \mu\right]} .
$$

Proof: See Appendix B.

The expressions of throughput for $\mathrm{SN}_{\mathrm{P}^{*}}$ and $\mathrm{SN}_{\mathrm{Q}}$ are formulated as,

$$
\begin{gathered}
\tau_{1}=\frac{\Omega\left(1-P_{\text {out }}^{s_{1}}\right)}{2} . \\
\tau_{2}=\frac{\Omega\left(1-P_{\text {out }}^{s_{2}}\right)}{2} .
\end{gathered}
$$

From (15)-(18), the closed-form expressions for $\mathrm{SN}_{\mathrm{P} *}$ and $\mathrm{SN}_{\mathrm{Q}}$ can be calculated, respectively.

\section{NUMERICAL RESULTS AND DISCUSSION}

We use the Monte Carlo simulation to verify the numerical results on the impact of key system parameters, i.e., transmit power, number of sensor nodes, on the system performance [21-25]. The primary simulation parameters are presented in Table 1. From Table 1, the distances of $\mathrm{SN}_{\mathrm{P} *}$ and $\mathrm{SN}_{\mathrm{Q}}$ to $\mathrm{R}$ and from $\mathrm{R}$ to $\mathrm{S}$ are calculated as follows:

Table 1. Simulation parameters

\begin{tabular}{ccc}
\hline Parameters & Notation & Typical Values \\
\hline Environment & & Rayleigh \\
Transmit power of sensor nodes & $\mathrm{P} 1, \mathrm{P} 2$ & $5,10 \mathrm{dBm}$ \\
Transmit power of the relay & $\mathrm{P} 3$ & $0-20 \mathrm{dBm}$ \\
The minimum required data rate & $\Omega$ & $1 \mathrm{bps} / \mathrm{Hz}$ \\
The coordinate of S & $\{\mathrm{x} 0, \mathrm{y} 0\}$ & $\{0,0\}$ \\
The coordinate of SNP* & $\{\mathrm{x} 1, \mathrm{y} 1\}$ & $\{1,0\}$ \\
The coordinate of SNQ & $\{\mathrm{x} 2, \mathrm{y} 2\}$ & $\{0,1\}$ \\
The coordinate of R & $\{\mathrm{x} 3, \mathrm{y} 3\}$ & $\{0.5,0.5\}$ \\
The path-loss exponent & $\theta$ & 2 \\
Number of sensor nodes of P & $\lambda$ & 1 \\
Number of sensor nodes of Q & $\mathrm{N}$ & $2,4,6$ \\
The parameters of the exponential distribution & $\mathrm{M}$ & 1 \\
The complexity-vs-accuracy trade-off coefficient & $\mathrm{L}$ & 100 \\
\hline
\end{tabular}

$$
\begin{aligned}
& d_{1}=\sqrt{\left(x_{1}-x_{3}\right)^{2}+\left(y_{1}-y_{3}\right)^{2}} \\
& d_{2}=\sqrt{\left(x_{2}-x_{3}\right)^{2}+\left(y_{2}-y_{3}\right)^{2}} \\
& d_{3}=\sqrt{\left(x_{0}-x_{3}\right)^{2}+\left(y_{0}-y_{3}\right)^{2}}
\end{aligned}
$$

Figure 2 depicts the outage probability $P_{\text {out }}$ versus the transmit power of relay by varying the transmit power of sensor nodes. As we observe from this Figure 2 that $P_{\text {out }}$ to detect $s_{1}$ and $s_{2}$ decreases when the transmit power increases. It means that the system performance can be improved by increasing the power at the relay. However, the impact of transmit power of sensor nodes on the system performance is quite different. Correctly, when increasing $P_{l}$ we can see that $P_{\text {out }}^{s_{1}}$ decreases and $P_{\text {out }}^{s_{2}}$ increases, on the contrary, $P_{\text {out }}^{s_{1}}$ increases and $P_{\text {out }}^{s_{2}}$ decreases when $P_{2}$ increases. It is explained that when increasing $P_{1}$ the interference affected the detection of $s_{2}$ at $\mathrm{S}$ is much more serious; thus, it makes $P_{\text {out }}^{s_{2}}$ increases. It is similar to the case of increasing $P_{2}$. 
Figure 3 depicts the flux of $\mathrm{SN}_{\mathrm{P} *}$ and $\mathrm{SN}_{\mathrm{Q}}\left(\tau_{1}\right.$ and $\left.\tau_{2}\right)$ versus $\mathrm{P}_{3}$ with different transmit power of sensor clusters. We see that $\tau_{1}$ and $\tau_{2}$ increase with increasing $\mathrm{P}_{3}$. It means that the performance of the system improves as the transmit power at the relay node $\mathrm{R}$ increases. Figure 3 also shows that when the transmit power of sensor cluster $\mathrm{P}$ decreases, the throughput $\tau_{1}$ decreases while the throughput $\tau_{2}$ increases. Furthermore, vice versa, when reducing the transmit power of sensor cluster $\mathrm{Q}$, the throughput $\tau_{2}$ decreases, while throughput $\tau_{1}$ increases.

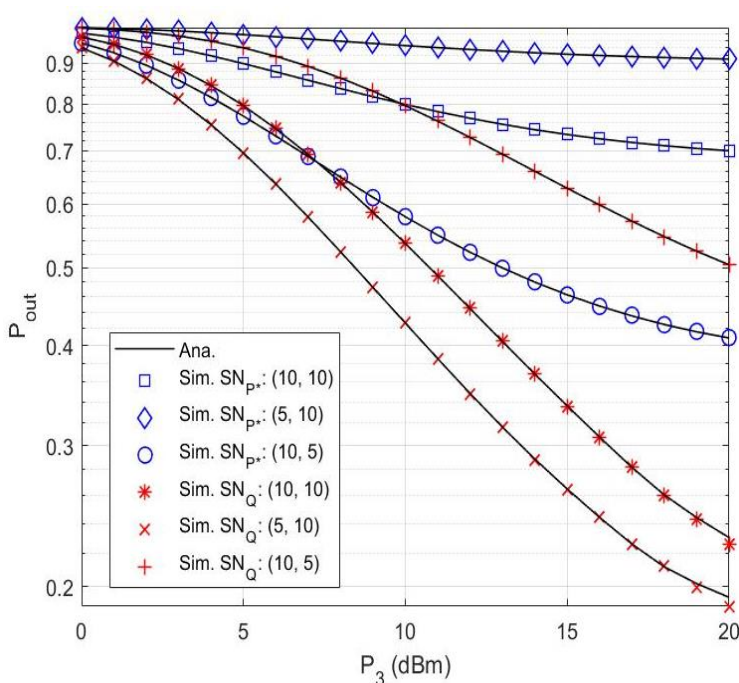

Figure 2. $\mathrm{P}_{\text {out }}$ versus the transmit power $\mathrm{P} 3$ with different $\mathrm{P}_{1}$ and $\mathrm{P}_{2}\left(\mathrm{P}_{1}, \mathrm{P}_{2}\right)$

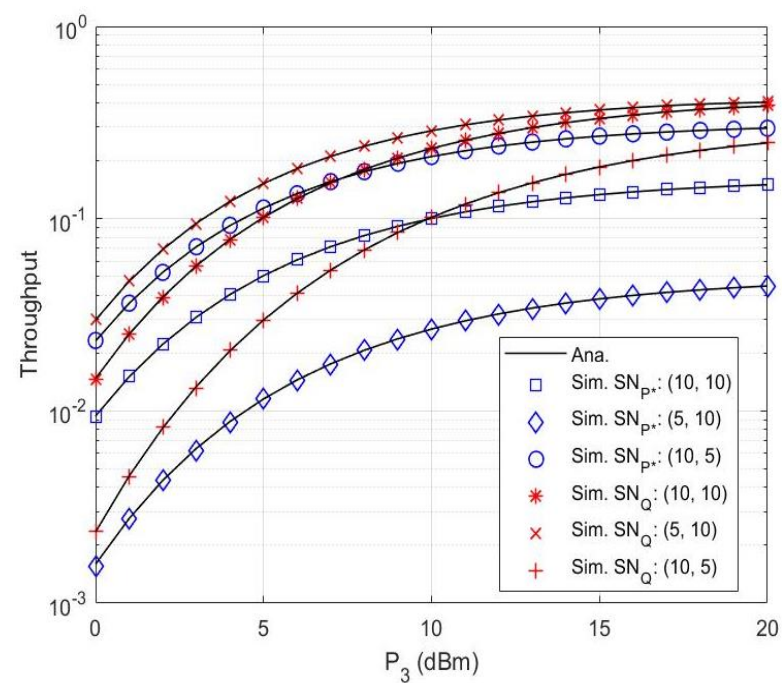

Figure 3. Throughput $\tau$ versus the transmit power $P_{3}$ with different $P_{1}$ and $P_{2}\left(P_{1}, P_{2}\right)$

Figure 4 and Figure 5 depict the effect of the number of sensor nodes at the sensor cluster $\mathrm{P}$ on the outage probability and throughput, respectively. The results once again confirm that when increasing the transmit power at $\mathrm{R}$, the system performance improves, as the $\mathrm{P}_{\text {out }}$ decreases and $\tau$ increases.

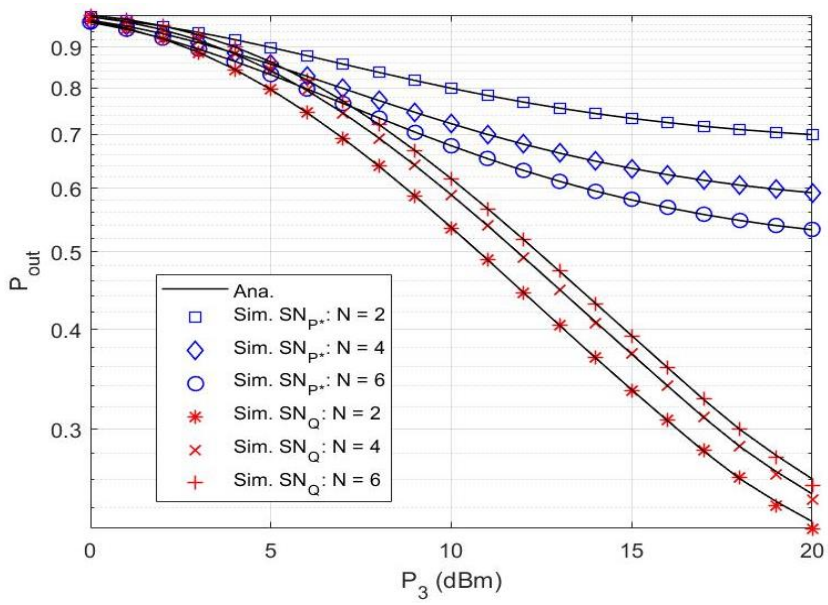

Figure 4. $\mathrm{P}_{\text {out }}$ versus the transmit power $\mathrm{P}_{3}$ with different $N$

Figure 4 draws the increase in the number of sensor nodes $N, P_{\text {out }}^{s_{1}}$ decreases, and $P_{\text {out }}^{s_{2}}$ increases. This can be explained as the more sensor nodes at $\mathrm{P}$, and the more likely the system is to find the best sensor node to carry out the data transmission of the sensor cluster P. The better the signal from the sensor cluster $\mathrm{P}$, the more interference it will generate for the sensor cluster $\mathrm{Q}$, resulting in $P_{\text {out }}^{s_{2}}$ increases. 
As shown in Figure 5, the number of sensor nodes $\mathrm{N}$ increases, $\tau 1$ increases, and $\tau 2$ decreases, meaning that the performance of the sensor cluster $\mathrm{P}$ is improved while the performance of the sensor cluster $\mathrm{Q}$ is worse. This conclusion is also consistent with the results obtained from Figure 5.

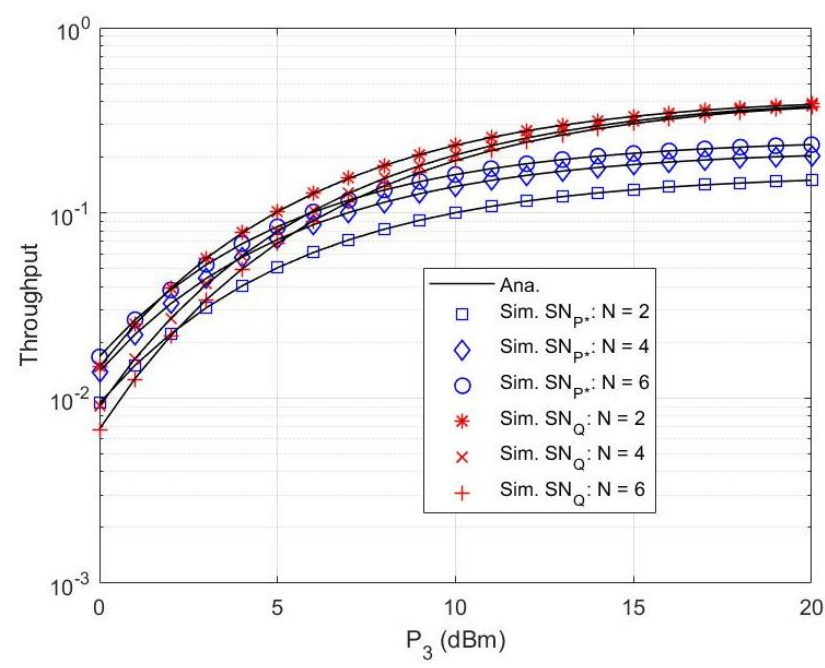

Figure 5. Throughput $\tau$ versus the transmit power $\mathrm{P}_{3}$ with different $\mathrm{N}$

\section{CONCLUSION}

In this work, we have investigated the multiuser uplink NOMA wireless sensor network with the best user selection scheme. The closed-form expressions of the system performance are derived. The results show that the system performance can be improved by increasing the transmit power at the relay. Increasing the number of sensor nodes at the sensor cluster $\mathrm{P}$ helps improve its performance but causes a decrease in the performance of the sensor cluster Q. The Monte Carlo simulation is conducted for validating the numerical results. In future studies, we will expand the system model with multi-relay.

\section{REFERENCES}

[1] Alam, M. M., Hamida, E. B., Rehmani, M., \& Pathan, A. "Wearable wireless sensor networks: Applications, standards, and research trends." In Emerging Communication Technologies Based on Wireless Sensor Networks: Current Research and Future Applications, pp. 59-88, 2016.

[2] Kurt, S., Yildiz, H. U., Yigit, M., Tavli, B., \& Gungor, V. C. "Packet size optimization in wireless sensor networks for smart grid applications." IEEE Transactions on Industrial Electronics, vol. 64, no.3, pp. 2392-2401, 2016.

[3] Noel, A. B., Abdaoui, A., Elfouly, T., Ahmed, M. H., Badawy, A., \& Shehata, M. S. "Structural health monitoring using wireless sensor networks: A comprehensive survey." IEEE Communications Surveys \& Tutorials, vol. 19, no. 3, pp. 1403-1423, 2017.

[4] Harb, H., Idrees, A. K., Jaber, A., Makhoul, A., Zahwe, O., \& Taam, M. A. "Wireless sensor networks: A big data source in Internet of Things.” International Journal of Sensors Wireless Communications and Control, vol. 7, no. 2, pp. 93-109, 2017

[5] Z. Zhang, H. Sun, and R. Q. Hu, "Downlink and uplink non-orthogonal multiple access in a dense wireless network," IEEE J. Sel. Areas Commun., vol. 35, no. 12, pp. 2771-2784, 2017.

[6] L. Dai, B. Wang, Y. Yuan, S. Han, C. I, and Z. Wang, "Non-orthogonal multiple access for 5G: solutions, challenges, opportunities, and future research trends," IEEE Commun. Mag., vol. 53, no. 9, pp. 74-81, 2015.

[7] Z. Ding, M. Peng, and H. V. Poor, "Cooperative non-orthogonal multiple access in 5G systems," IEEE Commun. Lett., vol. 19, no. 8, pp. 1462-1465, 2015.

[8] Hu, Z., Xu, L., Cao, L., Liu, S., Luo, Z., Wang, J., \& Wang, L. “Application of Non-Orthogonal Multiple Access in Wireless Sensor Networks for Smart Agriculture.” IEEE Access, vol. 7, pp. 87582-87592, 2019.

[9] Kim, B., Chung, W., Lim, S., Suh, S., Kwun, J., Choi, S., \& Hong, D. "Uplink NOMA with multi-antenna.” In 2015 IEEE 81 st vehicular technology conference (VTC Spring), pp. 1-5, 2019.

[10] Mouapi, A.; Hakem, N. A New Approach to Design Autonomous Wireless Sensor Node Based on RF Energy Harvesting System. Sensors 2018, 18, 133, doi:10.3390/s18010133.

[11] Liu, L., Sheng, M., Liu, J., Dai, Y., \& Li, J. "Stable Throughput Region and Average Delay Analysis of Uplink NOMA Systems With Unsaturated Traffic.” IEEE Transactions on Communications, vol. 67, no. 12, pp. 8475-8488, 2019. 
[12] Shin, W., Yang, H., Vaezi, M., Lee, J., \& Poor, H. V. "Relay-aided NOMA in uplink cellular networks." IEEE Signal Processing Letters, vol. 24, no. 12, pp. 1842-1846, 2017.

[13] Truong, V. T., Vo, M. T., Lee, Y., \& Ha, D. B. "Amplify-and-Forward Relay Transmission in Uplink NonOrthogonal Multiple Access Networks." In 2019 6th NAFOSTED Conference on Information and Computer Science (NICS), pp. 1-6, 2019.

[14] Tran, D. D., Ha, D. B., So-In, C., Tran, H., Nguyen, T. G., Baig, Z. A., \& Sanguanpong, S. " Performance Analysis of DF/AF Cooperative MISO Wireless Sensor Networks With NOMA and SWIPT Over Nakagami-\$ m \$ Fading." IEEE Access, vol. 6, pp. 56142-56161, 2018.

[15] Liu, H., Miridakis, N. I., Tsiftsis, T. A., Kim, K. J., \& Kwak, K. S. “ Coordinated uplink transmission for cooperative NOMA systems.” In 2018 IEEE Global Communications Conference (GLOBECOM,) pp. 1-6, 2018.

[16] Abdel-Razeq, S., Zhou, S., Bansal, R., \& Zhao, M. "Uplink NOMA transmissions in a cooperative relay network based on statistical channel state information." IET Communications, vol. 13, no. 4, pp. 371-378, 2018.

[17] Kader, M. F., Shin, S. Y., \& Leung, V. C. "Full-duplex non-orthogonal multiple access in cooperative relay sharing for 5G systems." IEEE Transactions on Vehicular Technology, vol. 67, no. 7, pp. 5831-5840, 2018.

[18] Lv, L., Chen, J., Ni, Q., Ding, Z., \& Jiang, H. "Cognitive non-orthogonal multiple access with cooperative relaying: A new wireless frontier for 5G spectrum sharing." IEEE Communications Magazine, vpl. 56, no. 4, pp. 188-195, 2018.

[19] Santamaria, P. Raimondo, M. Tropea, F. D. Rango, and C. Aiello, "An IoT Surveillance System Based on a Decentralised Architecture," Sensors, vol. 19, no. 6, p. 1469, 2019.

[20] P. Fazio, M. Tropea, F. Veltri, and S. Marano, "A Novel Rate Adaptation Scheme for Dynamic Bandwidth Management in Wireless Networks,” 2012 IEEE 75th Vehicular Technology Conference (VTC Spring), 2012. Zeng Hu, Longqin Xu, Liang Cao, Shuangyin Liu, Zhijie Luo, Jing Wang, Xiangli Li, Lu Wang. "Application of Non-Orthogonal Multiple Access in Wireless Sensor Networks for Smart Agriculture", IEEE Access, 2019.

[21] Duy-Hung Ha, Dac-Binh Ha, Jaroslav Zdralek, Miroslav Voznak. "Performance Analysis of Hybrid Energy Harvesting AF Relaying Networks over Nakagami-m Fading Channels", 2018 International Conference on Advanced Technologies for Communications (ATC), 2018.

[22] "Industrial Networks and Intelligent Systems", Springer Science and Business Media LLC, 2019.

[23] Van-Vinh Nguyen, Trong-Tuyen Tran, Vo Viet Tri, Van-Van Huynh, Hoang-Sy Nguyen, Miroslav Voznak. "Power-Splitting Protocol Non-Orthogonal Multiple Access (NOMA) in 5G Systems", Proceedings of the Tenth International Symposium on Information and Communication Technology - SoICT 2019, 2019.

[24] Dac-Binh Ha, Jai P. Agrawal. "Chapter 6 Performance Analysis for NOMA Relaying System in Next-Generation Networks with RF Energy Harvesting", Intech Open, 2020.

\section{APPENDIX}

APPENDIX A: Proof of Theorem 1.

Here, the expression of $P_{\text {out }}$ for $\mathrm{SN}_{\mathrm{P}^{*}}$ can be formulated as

$$
\begin{aligned}
& P_{\text {out }}^{s_{1}}=\operatorname{Pr}\left(\frac{\gamma_{1} \gamma_{3} Y_{1} X_{3}}{\left(\gamma_{2} X_{2}+1\right) \gamma_{3} X_{3}+\gamma_{1} Y_{1}+\gamma_{2} X_{2}+1}<\gamma_{t}\right) \\
& =\operatorname{Pr}\left\{\left[\gamma_{1} Y_{1}-\gamma_{t}\left(\gamma_{2} X_{2}+1\right)\right] \gamma_{3} X_{3}<\gamma_{t}\left(\gamma_{1} Y_{1}+\gamma_{2} X_{2}+1\right)\right\} \\
& =\operatorname{Pr}\left\{\gamma_{1} Y_{1}-\gamma_{t}\left(\gamma_{2} X_{2}+1\right)<0\right\}+\operatorname{Pr}\left\{X_{3}<\frac{\gamma_{t}\left(\gamma_{1} Y_{1}+\gamma_{2} X_{2}+1\right)}{\left[\gamma_{1} Y_{1}-\gamma_{t}\left(\gamma_{2} X_{2}+1\right)\right] \gamma_{3}}, \gamma_{1} Y_{1}-\gamma_{t}\left(\gamma_{2} X_{2}+1\right)>0\right\} \\
& =\operatorname{Pr}\left(\frac{\gamma_{1} Y_{1}}{\gamma_{2} X_{2}+1}<\gamma_{t}\right)+\operatorname{Pr}\left\{X_{3}<\frac{\gamma_{t}\left(\frac{\gamma_{1} Y_{1}}{\gamma_{2} X_{2}+1}+1\right)}{\gamma_{3}\left(\frac{\gamma_{1} Y_{1}}{\gamma_{2} X_{2}+1}-\gamma_{t}\right)}, \frac{\gamma_{1} Y_{1}}{\gamma_{2} X_{2}+1}>\gamma_{t}\right\} \\
& \stackrel{a}{=} 1+\sum_{i=1}^{N}\left(\begin{array}{c}
N \\
i
\end{array}\right)(-1)^{i} i \int_{\gamma_{t}}^{\infty} e^{-\frac{\gamma_{t}(u+1)}{\lambda_{3} \gamma_{3}\left(u-\gamma_{t}\right)}}\left[\frac{\lambda_{1} \gamma_{1} \lambda_{2} \gamma_{2}}{\left(\lambda_{1} \gamma_{1}+i \lambda_{2} \gamma_{2} u\right)^{2}}+\frac{1}{\lambda_{1} \gamma_{1}+i \lambda_{2} \gamma_{2} u}\right] e^{-\frac{i}{\lambda_{1} \gamma_{1}} u} d u \\
& =1+\sum_{i=1}^{N}\left(\begin{array}{c}
N \\
i
\end{array}\right)(-1)^{i} i \int_{0}^{\infty} e^{-\frac{\gamma_{t}\left(t+\gamma_{t}+1\right)}{\lambda_{3} \gamma_{3} t}}\left[\frac{\lambda_{1} \gamma_{1} \lambda_{2} \gamma_{2}}{\left(\lambda_{1} \gamma_{1}+i \lambda_{2} \gamma_{2}\left(t+\gamma_{t}\right)\right)^{2}}+\frac{1}{\lambda_{1} \gamma_{1}+i \lambda_{2} \gamma_{2}\left(t+\gamma_{t}\right)}\right] e^{-\frac{i\left(t+\gamma_{t}\right)}{\lambda_{1} \gamma_{1}}} d t \\
& \stackrel{b}{=} 1+\sum_{i=1}^{N}\left(\begin{array}{c}
N \\
i
\end{array}\right)(-1)^{i} \mu i e^{-\left(\frac{i}{\lambda_{1} \gamma_{1}}+\frac{1}{\lambda_{3} \gamma_{3}}\right) \gamma_{t}} \int_{0}^{1} \frac{\left(\lambda_{1} \gamma_{1} \lambda_{2} \gamma_{2} \ln z+\lambda_{1} \gamma_{1} \ln z+i \lambda_{2} \gamma_{2} \gamma_{t} \ln z-i \lambda_{2} \gamma_{2} \mu\right)}{\left(\lambda_{1} \gamma_{1} \ln z+i \lambda_{2} \gamma_{2} \gamma_{t} \ln z-i \lambda_{2} \gamma_{2} \mu\right)^{2} \ln z} e^{\frac{i \mu}{\lambda_{1} \gamma_{1} \ln z}} d z \\
& \stackrel{c}{=} 1+\sum_{i=1}^{N}\left(\begin{array}{c}
N \\
i
\end{array}\right)(-1)^{i} \frac{\pi \mu i e^{-\left(\frac{i}{\lambda_{1} \gamma_{1}}+\frac{1}{\lambda_{3} \gamma_{3}}\right) \gamma_{t}}}{2 L} \sum_{l=1}^{L}\left[\frac{\lambda_{1} \gamma_{1} \lambda_{2} \gamma_{2} \ln \frac{a_{l}+1}{2}+\lambda_{1} \gamma_{1} \ln \frac{a_{l}+1}{2}+i \lambda_{2} \gamma_{2} \gamma_{t} \ln \frac{a_{l}+1}{2}-i \lambda_{2} \gamma_{2} \mu}{\left(\lambda_{1} \gamma_{1} \ln \frac{a_{l}+1}{2}+i \lambda_{2} \gamma_{2} \gamma_{t} \ln \frac{a_{l}+1}{2}-i \lambda_{2} \gamma_{2} \mu\right)^{2} \ln \frac{a_{l}+1}{2}}\right] e^{\frac{i \mu}{\lambda_{1} \gamma_{1} \ln \frac{a_{l}+1}{2}}} \sqrt{1-a_{l}^{2}} .
\end{aligned}
$$


APPENDIX B: Proof of Theorem 2.

Here, the expression of $P_{\text {out }}$ for $\mathrm{SN}_{\mathrm{Q}}$ can be calculated by

$$
\begin{aligned}
& P_{\text {out }}^{s_{2}}=\operatorname{Pr}\left(\frac{\gamma_{2} \gamma_{3} X_{2} X_{3}}{\gamma_{1} Y_{1}+\gamma_{2} X_{2}+\gamma_{3} X_{3}+1}<\gamma_{t}\right) \\
& =F_{X_{2}}\left(\frac{\gamma_{t}}{\gamma_{2}}\right)+\int_{0}^{\infty} \int_{\frac{\gamma_{t}}{\gamma_{2}}}^{\infty} F_{X_{3}}\left(\frac{\gamma_{t}\left(\gamma_{1} y+\gamma_{2} x+1\right)}{\gamma_{3}\left(\gamma_{2} x-\gamma_{t}\right)}\right) f_{X_{2}}(x) d y f_{Y_{1}}(y) d x \\
& =1-\sum_{i=0}^{N-1}\left(\begin{array}{c}
N-1 \\
i
\end{array}\right)(-1)^{i} \int_{0}^{\infty} \int_{0}^{\infty} \frac{e^{-\frac{\gamma_{t}\left(\gamma_{1} y+t+\gamma_{t}+1\right)}{\lambda_{3} \gamma_{3} t}-\frac{t+\gamma_{t}}{\lambda_{2} \gamma_{2}}-\frac{(i+1) y}{\lambda_{1}}}}{\lambda_{1} \lambda_{2}} d y d t \\
& =1-\sum_{i=0}^{N-1}\left(\begin{array}{c}
N-1 \\
i
\end{array}\right)(-1)^{i} \int_{0}^{\infty} \frac{e^{-\frac{\gamma_{t}\left(t+\gamma_{t}+1\right)}{\lambda_{3} \gamma_{3} t}-\frac{t+\gamma_{t}}{\lambda_{2} \gamma_{2}}}}{\lambda_{1} \lambda_{2}} \int_{0}^{\infty} e^{-\left(\frac{\gamma_{1} \gamma_{1}}{\lambda_{3} \gamma_{3} t}+\frac{i+1}{\lambda_{1}}\right) y} d y d t \\
& =1-\sum_{i=0}^{N-1}\left(\begin{array}{c}
N-1 \\
i
\end{array}\right)(-1)^{i} e^{-\left(\frac{1}{\lambda_{2} \gamma_{2}}+\frac{1}{\lambda_{3} \gamma_{3}}\right) \gamma_{1}^{\infty}} \int_{0}^{\infty} \frac{\lambda_{3} \gamma_{3} t}{\lambda_{2}\left[\lambda_{1} \gamma_{1} \gamma_{t}+(i+1) \lambda_{3} \gamma_{3} t\right]} e^{-\frac{\gamma_{t}\left(\gamma_{t}+1\right)}{\lambda_{3} \gamma_{3} t}-\frac{t}{\lambda_{2} \gamma_{2}}} d t \\
& =1-\sum_{i=0}^{N-1}\left(\begin{array}{c}
N-1 \\
i
\end{array}\right)(-1)^{i} e^{-\left(\frac{1}{\lambda_{2} \gamma_{2}}+\frac{1}{\lambda_{3} \gamma_{3}}\right) \gamma_{t}} \int_{0}^{1} \frac{-\lambda_{3} \gamma_{3} \mu^{2}}{\lambda_{2}\left[\lambda_{1} \gamma_{1} \gamma_{t} \ln z-(i+1) \lambda_{3} \gamma_{3} \mu\right] \ln ^{2} z} e^{\frac{\mu}{\lambda_{2} \gamma_{2} \ln z}} d z \\
& =1-\frac{\pi \mu^{2} N \lambda_{3} \gamma_{3}}{2 L \lambda_{2} \gamma_{2}} e^{-\left(\frac{1}{\lambda_{2} \gamma_{2}}+\frac{1}{\lambda_{3} \gamma_{3}}\right) \gamma_{t}} \sum_{i=0}^{N-1}\left(\begin{array}{c}
N-1 \\
i
\end{array}\right)(-1)^{i} \sum_{l=1}^{L} \frac{e^{\frac{\mu}{\lambda_{2} \gamma_{2} \ln \frac{a_{l}+1}{2}}} \sqrt{1-a_{l}^{2}}}{\ln ^{2} \frac{a_{l}+1}{2}\left[\lambda_{1} \gamma_{1} \gamma_{t} \ln \frac{a_{l}+1}{2}-(i+1) \lambda_{3} \gamma_{3} \mu\right]} .
\end{aligned}
$$

